\title{
Temporary Stent-Assisted Coil Embolization as a Treatment Option for Wide-Neck Aneurysms
}

\author{
(D) M. Müller, (D) B. Brockmann, DS. Afat, (D) Nikoubashman, (D) G.A. Schubert, (D)A. Reich, (D)A.E. Othman, and (D). Wiesmann
}

\begin{abstract}
BACKGROUND AND PURPOSE: Simple coil embolization is often not a feasible treatment option in wide-neck aneurysms. Stent-assisted coil embolization helps stabilize the coils within the aneurysm. Permanent placement of a stent in an intracranial vessel, however, requires long-term platelet inhibition. Temporary stent-assisted coiling is an alternative technique for the treatment of wide-neck aneurysms. To date, only case reports and small case series have been published. Our purpose was to retrospectively analyze the effectiveness and safety of temporary stent-assisted coiling in a larger cohort.
\end{abstract}

MATERIALS AND METHODS: Research was performed for all patients who had undergone endovascular aneurysm treatment in our institution (University Hospital Aachen) between January 2010 and December 2015. During this period, 355 consecutive patients had undergone endovascular aneurysm treatment. We intended to treat $33(9.2 \%)$ of them with temporary stent-assisted coiling, and they were included in this study. Incidental and acutely ruptured aneurysms were included.

RESULTS: Sufficient occlusion was achieved in $97.1 \%$ of the cases. In $94 \%$, the stent could be fully recovered. Complications occurred in 5 patients (14.7\%), whereas in only 1 case was the complication seen as specific to stent-assisted coiling.

CONCLUSIONS: Temporary stent-assisted coiling is an effective technique for the treatment of wide-neck aneurysms. Safety is comparable with that of stent-assisted coiling and coiling with balloon remodeling.

S imple coil embolization is often not a feasible treatment option in wide-neck aneurysms. Stent-assisted coil embolization helps stabilize the coils within the aneurysm. ${ }^{1}$ Permanent placement of a stent in an intracranial vessel, however, requires longterm platelet inhibition. Platelet inhibition is known to be associated with a higher bleeding risk. ${ }^{2}$ Particularly in patients who require further treatment in an intensive care unit, such as patients with an acute subarachnoid hemorrhage, a higher bleeding risk should be avoided. In addition, dual platelet inhibition as recommended when stents are deployed permanently, increases the risk for cerebral hemorrhage within potentially existent isch-

Received October 17, 2016; accepted after revision February 22, 2017.

From the Departments of Neuroradiology (M.M., C.B., S.A., O.N., M.W.), Neurosurgery (G.A.S.), and Neurology (A.R.), University Hospital Aachen, Aachen, Germany; Institute of Neuroscience and Medicine 4 (O.N.), Forschungszentrum Jülich GmbH Jülich, Germany; and Department for Diagnostic and Interventional Radiology (A.E.O.), Eberhard Karls University Tuebingen, University Hospital Tuebingen, Tübingen, Germany.

Please address correspondence to Marguerite Müller, MD, Department of Neuroradiology, University Hospital Aachen, Pauwelsstr 30, 52074 Aachen, Germany;

e-mail: mamueller@ukaachen.de

http://dx.doi.org/10.3174/ajnr.A5204 emic brain tissue due to vasospasms. ${ }^{3}$ Hence, avoiding permanent stent placement is an advantage in patients with acute $\mathrm{SAH}$.

In the past, several techniques have been established for the treatment of wide-neck aneurysms. Common techniques are stent-assisted coiling, balloon-assisted coiling, double microcatheter coiling, and aneurysm treatment with dedicated devices such as the Woven EndoBridge (WEB; Sequent Medical, Aliso Viejo, California) device ${ }^{4,5}$ or the Comaneci device (Rapid Medical, Yokneam, Israel). ${ }^{6,7}$ The combination of balloon remodeling and stent-assisted coiling is another treatment option with possible achievement of higher occlusion rates. ${ }^{8}$

Stent-assisted coiling and balloon remodeling seem to have a comparable complication rate in the literature, ranging between $10 \%$ and $20 \% .^{9,10}$

Treatment of wide neck-aneurysms is also possible with flow diverter devices with an occlusion rate of about $80 \% .{ }^{11}$ However, with a latency of 4-12 months to aneurysm thrombosis and the need for subsequent platelet inhibition, these are not a primary option in patients with an acutely ruptured intracranial aneurysm.

Another treatment option for wide-neck aneurysms is temporary stent-assisted coiling. ${ }^{12,13}$ For this purpose, 2 microcatheters 

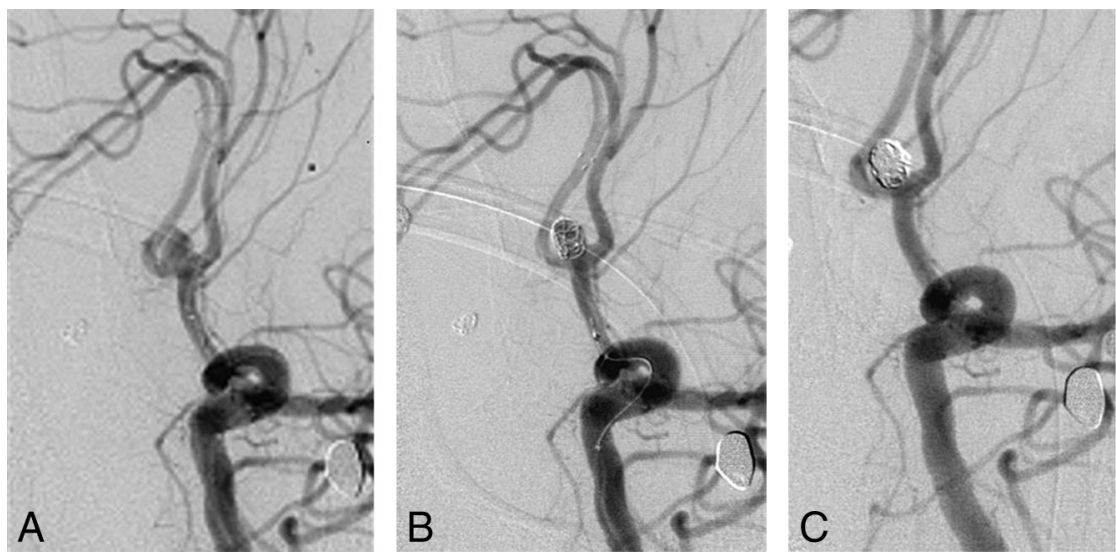

FIG 1. A, An aneurysm of the anterior communicating artery before coiling. $B$, The same aneurysm partially coiled with a deployed Solitaire stent from the left $A 1$ to the right $A 2$ segment. $C$, The same aneurysm after complete coil embolization. The Solitaire stent has been recovered.
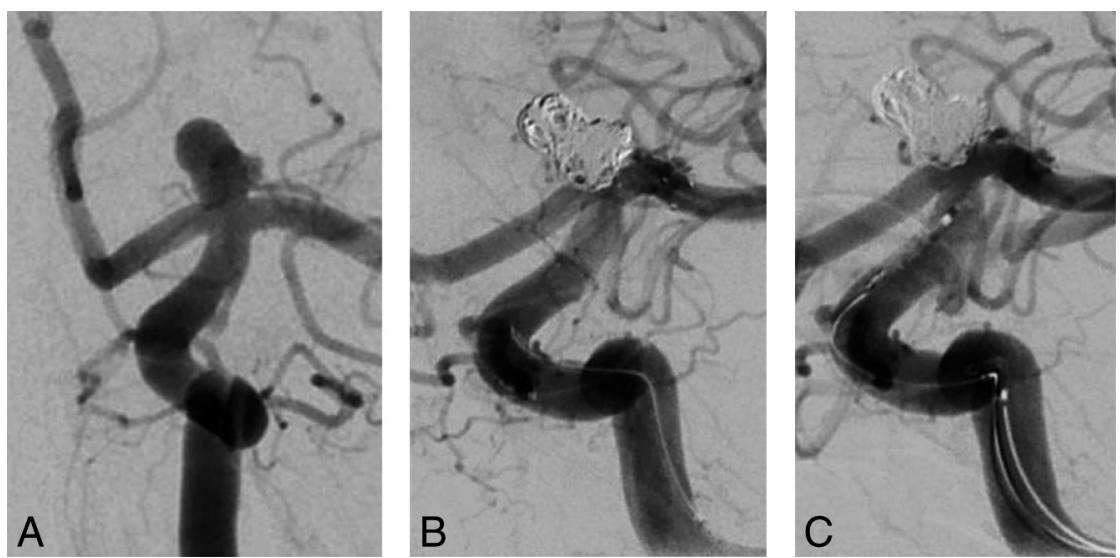

FIG 2. A, A carotid-T aneurysm before coiling. $B$, The same aneurysm partially coiled with the deployed Solitaire stent from the internal carotid artery to the left M1 segment. C, The same aneurysm after complete coil embolization. The Solitaire stent has been recovered. dergone endovascular aneurysm treatment in our institution (University Hospital Aachen) between January 2010 and December 2015. During this period, 355 consecutive patients had undergone endovascular aneurysm treatment. We intended to treat $33(9.2 \%)$ of them with temporary stent-assisted coiling, and they were included in this study. In the same period, 4 (1.1\%) patients were treated with balloon remodeling. Incidental and acutely ruptured aneurysms were included.

\section{Clinical and Procedural Data}

Evaluation concerned the possibility of stent recovery after coiling, occlusion rate and recurrence of the aneurysm, and the procedure-related complication rate.

Aneurysm occlusion was determined by using the modified Raymond-Roy classification. ${ }^{15}$

We also noted the location and the maximal diameter of the aneurysm and the dome-to-neck ratio and the diameter of the parent vessel. Peri-interventional and posttreatment platelet inhibition management was recorded for all patients.

\section{Statistical Analysis}

Correlations between variables were calculated by using Pearson ProductMoment Correlation Coefficient. $P$ values $\leq .05$ were significant. All statistical are used. The first microcatheter is used to cover the aneurysm neck and deploy the stent. The second microcatheter is advanced into the aneurysm to perform coil embolization. Advancement of the second microcatheter into the aneurysm can be performed either before deployment of the stent or after stent deployment. ${ }^{14}$ The stent is deployed to cover the aneurysm neck but is not fully released. After coil embolization has been completed, the stent is recovered (Figs 1 and 2). In the unlikely event of coil protrusion during the process of recovery of the stent, recovery is stopped, the stent is deployed again, and it is released for permanent implantation. Temporary stent-assisted coiling is an established standard technique in our institution for the treatment of wideneck aneurysms. Because to date, only case reports and small case series have been published, we analyzed a larger cohort of patients treated with temporary stent-assisted coiling. ${ }^{12,13}$ Following, we present a retrospective analysis on the effectiveness and safety of temporary stent-assisted coiling.

\section{MATERIALS AND METHODS \\ Patients}

After approval from our local ethics committee, we retrospectively searched our clinical data base for all patients who had un- analyses were performed with SPSS 23 software (IBM, Armonk, New York).

\section{RESULTS}

Thirty-three patients ( 25 women) with 34 aneurysms, with the intention to treat by temporary stent-assisted coiling, were included in the retrospective analysis. Twenty of the treated aneurysms were acutely ruptured, 2 were symptomatic but unruptured, and 12 were incidental. Ages ranged from 29 to 79 years with a mean of $55 \pm 12$ years.

Fifteen aneurysms were located at the anterior communicating artery; 5 , at the basilar tip; 11, at the carotid artery (with 6 of these located at the carotid-T); 1 , at the middle cerebral artery bifurcation; 1, at the posterior communicating artery; and 1, at the origin of the posterior inferior cerebellar artery.

Maximal aneurysm diameters were 3-18 $\mathrm{mm}$, with a mean maximal diameter of $7 \pm 3.4 \mathrm{~mm}$. Parent vessel diameters were 0.9-4.4 mm, with a mean of $2.1 \pm 0.75 \mathrm{~mm}$. Dome-to-neck ratios were $0.8-3.33$, with a mean of $1.44 \pm 0.55$ and a median of 1.33 .

For temporary stent placement, a Solitaire AB stent (Covidien, Irvine, California) was used in 30 cases. In 3 cases, an Enterprise stent (Codman \& Shurtleff, Raynham, Massachusetts) was used, 
and 1 aneurysm was treated with a LEO stent (Balt Extrusion, Montmorency, France).

Concerning initial treatment success, 26 aneurysms were completely occluded (class I), 7 aneurysms had a residual dog-ear (class II), and in 1 aneurysm, there was a noted a residuum of the aneurysm (class IIIb). Hence, the complete occlusion rate was $76.5 \%$, and a clinically acceptable occlusion rate, including a dogear remnant, was $97.1 \%$. Statistical analysis showed no significant correlation between the occlusion rate and diameters of the aneurysm $(P=.50)$ or the parent artery $(P=.13)$ and no significant correlation to the dome-to-neck ratio $(P=.19)$.

For 16 patients, follow-up MR imaging or digital subtraction angiography was available for at least 6 months. Three of 16 had an aneurysm recurrence, and 13 were stable during follow-up, with no significant correlation between recurrence and the diameters of the aneurysm $(P=.30)$ or the parent artery $(P=.77)$ and no significant correlation to the dome-to-neck ratio $(P=.97)$.

For 9 patients, follow-up data were available for at least 2 years. Two of $9(22.2 \%)$ had an aneurysm recurrence, and 7 were stable during long-term follow up.

In 2 of the 34 aneurysms, it was not possible to safely recover the stent, due to impending coil migration into the parent artery. Hence, the overall success rate of stent recovery was $94 \%$. Statistical analysis showed no significant correlation between successful stent recovery and the diameters of the aneurysm $(P=.88)$ or the parent artery $(P=.54)$ and no significant correlation to the dome-to-neck ratio $(P=.52)$.

All patients were treated with platelet inhibition during the procedure with 28 patients receiving intravenous tirofiban, 2 patients receiving IV acetyl salicylic acid, and 3 patients being pretreated with dual platelet inhibition (acetyl salicylic acid and clopidogrel). Pretreatment with antiplatelet drugs in those patients was due to preexisting conditions; 2 had coronary heart disease, and in 1 patient, another aneurysm had previously been treated with stent-assisted coiling.

Posttreatment, 1 of the 2 patients with permanent stent placement was given dual platelet inhibition for 3 months. The other patient with permanent stent placement received mono antiplatelet therapy with acetyl salicylic acid for 3 months. Both patients had acutely ruptured aneurysms.

Concerning the patients with stent recovery, 8 patients ( 3 with an acutely ruptured aneurysms) were given dual platelet inhibition for 4 weeks to 3 months. Five patients ( 3 with an acutely ruptured aneurysm) were given a single dose of $500 \mathrm{mg}$ of acetyl salicylic acid subsequent to the procedure. Fourteen patients ( 8 with an acutely ruptured aneurysm) received mono antiplatelet therapy for 10 days to 6 months. Five patients ( 4 with an acutely ruptured aneurysms) were not given any subsequent antiplatelet medication.

Periprocedural complications occurred in 5 patients (14.7\%). In the first patient, stretching and tearing of a coil occurred. During the process of retrieving the coil, there was thrombus formation in the afferent vessel that was completely resolved by IV rtPA and mechanical recanalization with a Solitaire AB 4/20 (ev3 Neurovascular, Irvine, California). Peri-interventional antiplatelet therapy was performed with tirofiban. The modified Rankin Scale score at discharge was 1 .
In the second patient, no peri-interventional emboli were detected, yet postinterventional MR imaging showed multiple emboli in the vascular territory of the parent artery. Peri-interventional antiplatelet therapy was performed with tirofiban. The mRS at discharge was 1 , due to a residual pronation.

In the third patient, again no peri-interventional emboli were detected with scattered bi-hemispheric ischemic lesions on postinterventional MR imaging. Peri-interventional antiplatelet therapy was performed with tirofiban. The mRS at discharge was 4 , due to hemiparesis. With bi-hemispheric lesions and atrial fibrillation, in this case thromboembolism might also have been of cardiac origin.

In the fourth patient, first an occlusion of the stent lumen and perforation of the right ICA occurred. This complication was managed by an occlusion of the right ICA. Peri-interventional antiplatelet therapy was performed with tirofiban. The mRS at discharge was 5 .

In the fifth patient, peri-interventional perforation of the aneurysm occurred during coil deployment. This was quickly managed by further coil embolization. Peri-interventional antiplatelet therapy was administered with tirofiban. The mRS at discharge was 3.

In summary, 4 of these 5 cases were due to thromboembolic events ( 3 with a Solitaire AB stent and 1 with an Enterprise stent), whereas the remaining complication was due to perforation of the aneurysm during coil deployment. Stent occlusion may be a complication related to the specific procedure, whereas the other complications are the usual ones observed in any technique of aneurysm embolization. There was a moderate negative correlation between the complication rate and dome-to-neck ratio (Pearson Product-Moment Correlation Coefficient $=-0.38, P=.026$ ).

Concerning the 4 patients treated with balloon remodeling, 3 aneurysms were located on the internal carotid artery and 1 was of the basilar tip. One was a symptomatic aneurysm, and the other 3 were incidental. None of the aneurysms treated with balloon remodeling were ruptured.

Complete occlusion was achieved in 2 cases: In 1 case, a dogear remained, and in 1 case, there was a remaining perfusion of the aneurysm.

Two patients were pretreated with dual platelet inhibition (acetyl salicylic acid and clopidogrel) given for 3 months posttreatment. Two patients received tirofiban during the procedure: One was treated with dual platelet inhibition (acetyl salicylic acid and clopidogrel) for 3 months posttreatment, and 1 received only acetyl salicylic acid for 6 months.

Follow-up data were available for 3 patients for at least 12 months, with 1 recurrence of the aneurysm that had not been completely occluded.

There was 1 complication with a cerebral infarction. In this case, peri-interventional treatment was performed with tirofiban.

Statistical comparison was not performed due to the small sample size in the group treated with balloon remodeling.

\section{DISCUSSION}

Our results show that in this small study, temporary stent-assisted coiling is a feasible and effective option for the treatment of wideneck aneurysms. This technique has been described in previous literature using fully retrievable stents such as the Solitaire 
AB. ${ }^{12,13}$ The number of cases in those studies was 3 and 8 , respectively. Therefore, this is the first report of a larger collective. Furthermore, we were able to show that this technique is also possible with the Enterprise and LEO stents if not completely deployed during the procedure.

In summary, our results imply that effective stent-assisted coiling with incomplete deployment of a variety of stents can be achieved with a satisfactory complication rate. It was possible to achieve a clinically acceptable occlusion rate (including a dog-ear remnant) in 33/34 (97.1\%) cases and to recover the stent after completion of coiling with a success rate of $94 \%$, regardless of the dome-to-neck ratio. Concerning the immediate effectiveness of temporary stent-assisted coiling, we believe a complete occlusion rate of $76.5 \%$ (class $\mathrm{I})^{15}$ and an occlusion rate including dog-ear remnants (classes I + II) of $97.1 \%$ to be rather satisfying and comparable with a previous study that also indicates that a complete occlusion is more likely with stent assistance rather than balloon remodeling. ${ }^{16}$

A recurrence rate of $18.8 \%$ at 6 months' follow-up and $22.2 \%$ at long-term follow-up is comparable with previously mentioned recurrence rates of wide-neck aneurysms treated by stent-assisted coiling or balloon remodeling. ${ }^{17}$ Yet, with a higher sample size and more follow-up results, the recurrence rate might be lower because a high occlusion rate is thought to result in a smaller recurrence rate. ${ }^{18}$ However, with a sample size of only 16 patients with an available follow-up of at least 6 months, we believe that a safe assumption concerning the long-term results cannot be made with this analysis.

The overall complication rate of $14.7 \%$ is comparable with that of stent-assisted coiling with permanent stent placement and balloon remodeling, in which complication rates ranging between $10 \%$ and $20 \%$ have been reported. ${ }^{9}$ Because most complications were caused by thromboembolic events, we believe that using a sufficient platelet inhibition and an anticoagulation regimen during the procedure with a partially deployed stent is mandatory, despite removal at the end of the procedure. On the basis of a complication rate not higher than that of stent-assisted coiling, ${ }^{9}$ we believe that there is no higher risk involved in an incomplete deployment of the device during the procedure and there does not seem to be a difference between the devices used in our analysis.

Our data, however, do not allow determining whether and how long subsequent (long-term) platelet inhibition is necessary. Posttreatment antiplatelet therapy varied considerably within our patient group. This variation is because in each individual case, the neurointerventionalist has to consider a variety of factors including, for example, the presence of acute hemorrhage, the expected propensity of the coil/parent vessel interface to develop thrombus, surgical procedures potentially required such as CSF drainage, and a variety of other medical conditions. Even though there were no delayed embolisms in the 5 patients who did not receive subsequent platelet inhibition, the relatively small sample size of our study does not allow a valid recommendation. Yet, it seems reasonable that subsequent platelet inhibition should be performed as is common in cases with aneurysms treated only by coiling that has a broad interface with the parent vessel. Therefore, in patients with an unruptured intracranial aneurysm, subsequent platelet inhibition may be reasonable, whereas in patients with an impending bleeding risk such as those with an acute SAH, further platelet inhibition should not be enforced.

Overall, we believe that temporary stent-assisted coiling is an alternative to balloon remodeling with the advantages of an easier navigation in smaller tortuous vessels and the absence of temporary flow arrest in the parent artery. ${ }^{19}$

The limitations of our study are a still rather small sample size and a possible selection bias because the patients were not prospectively randomized to the different treatment techniques for wide-neck aneurysms.

\section{CONCLUSIONS}

In this small study, temporary stent-assisted coiling is an effective technique for the treatment of wide-neck aneurysms. Safety is comparable with that of stent-assisted coiling and coiling with balloon remodeling. The major advantage of this treatment is less need for subsequent platelet inhibition, which might cause cerebral hemorrhage or complicate further treatment in an intensive care unit, and is therefore particularly eligible for patients with an acutely ruptured intracranial aneurysm.

Disclosures: Martin Wiesmann—UNRELATED: Consultancy: Stryker Neurovascular: Payment for Lectures Including Service on Speakers Bureaus: Bracco, Siemens, Stryker Neurovascular; Royalties: Springer, Thieme; Payment for Development of Educational Presentations: Abbott Laboratories, ab medica, Acandis, Bayer, Bracco, B. Braun, Codman Neurovascular, Covidien, Dahlhausen, MicroVention, Penumbra, phenox, Philips Healthcare, Siemens, Silk Road Medical, St. Jude Medical, Stryker Neurovascular.* *Money paid to the institution.

\section{REFERENCES}

1. Gory B, Klisch J, Bonafé A, et al. Solitaire AB stent-assisted coiling of wide-necked intracranial aneurysms: mid-term results from the SOLARE study. Neurosurgery 2014;75:215-19; discussion 219 CrossRef Medline

2. Aradi D, Kirtane A, Bonello L, et al. Bleeding and stent thrombosis on P2Y12-inhibitors: collaborative analysis on the role of platelet reactivity for risk stratification after percutaneous coronary intervention. Eur Heart J 2015;36:1762-71 CrossRef Medline

3. Geraghty OC, Kennedy J, Chandratheva A, et al. Preliminary evidence of a high risk of bleeding on aspirin plus clopidogrel in aspirin-naïve patients in the acute phase after TIA or minor ischaemic stroke. Cerebrovasc Dis 2010;29:460-67 CrossRef Medline

4. Behme D, Berlis A, Weber W. Woven EndoBridge intrasaccular flow disrupter for the treatment of ruptured and unruptured wide-neck cerebral aneurysms: report of 55 cases. AJNR Am J Neuroradiol 2015; 36:1501-06 CrossRef Medline

5. Pierot L, Spelle L, Molyneux A, et al. Clinical and anatomical follow-up in patients with aneurysms treated with the WEB device: 1-year follow-up report in the cumulated population of 2 prospective, multicenter series (WEBCAST and French Observatory). Neurosurgery 2016;78:133-41 CrossRef Medline

6. Lawson AL, Chandran A, Puthuran M, et al. Initial experience of coiling cerebral aneurysms using the new Comaneci device. BMJ Case Rep 2015;2015:pii: bcr2015011726 CrossRef Medline

7. Gupta R, Kolodgie FD, Virmani R, et al. Comaneci neck bridging device for the treatment of cerebral aneurysms. J Neurointerv Surg 2016;8:181-85 CrossRef Medline

8. Gentric JC, Biondi A, Piotin M, et al. Balloon remodeling may improve angiographic results of stent-assisted coiling of unruptured intracranial aneurysms. Neurosurgery 2015;76:441-45; discussion 445 CrossRef Medline

9. Chitale R, Chalouhi N, Theofanis $\mathrm{T}$, et al. Treatment of ruptured intracranial aneurysms: comparison of stenting and balloon remodeling. Neurosurgery 2013;72:953-59 CrossRef Medline

AJNR Am J Neuroradiol 38:1372-76 Jul 2017 www.ajnr.org 
10. Moon K, Albuquerque FC, Ducruet AF, et al. Balloon remodeling of complex anterior communicating artery aneurysms: technical considerations and complications. J Neurointerv Surg 2015;7: 418-24 CrossRef Medline

11. Yakovlev SB, Arustamyan SR, Dorokhov PS, et al. Endovascular treatment of large and giant intracranial aneurysms using flow-diverting stents [Article in Russian; Abstract available in Russian from the publisher]. Zh Vopr Neirokhir Im N N Burdenko 2015;79:19-27 Medline

12. Almekhlafi MA, Hockley A, Wong JH, et al. Temporary Solitaire stent neck remodeling in the coiling of ruptured aneurysms. J Neurointerv Surg 2013;5(suppl 3):iii76-78 CrossRef Medline

13. Signorelli F, Gory B, Turjman F. Temporary Solitaire stent-assisted coiling: a technique for the treatment of acutely ruptured wideneck intracranial aneurysms. AJNR Am J Neuroradiol 2014;35: 984-88 CrossRef Medline

14. Spiotta AM, Wheeler AM, Smithason S, et al. Comparison of techniques for stent assisted coil embolization of aneurysms. J Neurointerv Surg 2012;4:339-44 CrossRef Medline
15. Stapleton CJ, Torok CM, Rabinov JD, et al. Validation of the Modified Raymond-Roy classification for intracranial aneurysms treated with coil embolization. J Neurointerv Surg 2016;8:927-33 CrossRef Medline

16. Consoli A, Vignoli C, Renieri L, et al. Assisted coiling of saccular wide-necked unruptured intracranial aneurysms: stent versus balloon. J Neurointerv Surg 2016;8:52-57 CrossRef Medline

17. Peterson E, Hanak B, Morton R, et al. Are aneurysms treated with balloon-assisted coiling and stent-assisted coiling different? Morphological analysis of 113 unruptured wide-necked aneurysms treated with adjunctive devices. Neurosurgery 2014;75:145-51; quiz 151 CrossRef Medline

18. Murias Quintana E, Gil Garcia A, Vega Valdes P, et al. Anatomical results, rebleeding and factors that affect the degree of occlusion in ruptured cerebral aneurysms after endovascular therapy. J Neurointerv Surg 2015;7:892-97 CrossRef Medline

19. Zumofen DW, Sahasrabudhe N, Riina HA, et al. Temporary stent scaffolding during aneurysm coiling. JClin Neurosci 2014;21:852-54 CrossRef Medline 